\title{
CARDINAL PREFERENCE AND STRUCTURE IN DECISION MAKING
}

\author{
Brady M. Cillo \\ United States Air Force \\ 733 S. Negley Ave \#1 \\ Pittsburgh, PA 15232 \\ brady.cillo@gmail.com
}

Keywords: ANP, cardinal preference, structure, good and bad

\begin{abstract}
Summary: When the consequences of a decision are high, one would expect the decision making process to be very meticulous and structured. However, many complex decisions get filtered with the traditional processes used for everyday decisions. Common flaws such as relying on gut feelings or the power of the mind alone inhibit good decision making. Most inherent to decisions is the lack of ability to quantify emotions into anything more than ordinal preferences. The Analytic Network Process (ANP) overcomes this basic flaw by offering methods of quantifying intangible judgments. The ability to output cardinal preference is fundamental to ANP, but further structure is required to tie complex decisions together. ANP provides both positive and negative decision networks and incorporates complex relationships into the process. These characteristics are illustrated through the use of examples compiled over the past four years done by professionals from around the world.
\end{abstract}

\section{Introduction}

People make countless decisions all through the day. The decisions can be mundane, such as, "When should I wake up?", “Should I wear the red shirt or the blue shirt?", or "When should I brush my teeth, and for how long?" Other decisions come more infrequently and thus greater consideration is taken in determining an answer: "Which bus route takes me downtown most quickly?", "Who can baby-sit our children?”, or "How much money should I allocate to each retirement fund?”

At every juncture of the day, we make decisions at both the conscious and subconscious level. The results of most decisions are inconsequential. The red shirt brings no more satisfaction than the blue shirt. But when the consequences of a decision carry more weight, does our decision making process change? Consider the option of buying either a TV dinner or filet mignon for dinner. There are obvious price differences between the two selections, but that may not be the only factor in the decision. Perhaps money is not an issue for the person, but time is a valid constraint. Maybe he's just moved into town and doesn't yet have a grill, but does have a microwave. Or perhaps dietary restrictions cause one option to appear more favorable. Even though the options are limited, the decision criteria can quickly become overwhelming. How can one ensure a sound decision with so many variables, all while standing in aisle 10 of the grocery store?

The ramifications of the dinner selection can only produce temporary satisfaction or dissatisfaction. When decisions become several magnitudes larger, long-run consequences can eclipse both the decision making process and short-run factors. Businesses and governments are often faced with situations where their very state of existence depends on sound decisions. Billions of dollars or millions of lives definitely raise the stakes of these outcomes.

Thus, when the consequences of a decision are high, one would expect the decision making process to be very meticulous and structured. Unfortunately, the same features that plague simplistic decisions still manage to hinder decisions of a grander scale. First and foremost, the nature of language and emotions tends to produce ordinal solutions that do not effectively quantify one's true feelings and preferences. Individuals may prefer one option to another, but are unable to convey why or by how much. The lack of cardinal preferences is perhaps the single greatest inhibitor to sound decision making. 
Besides the tendency for ordinal preferences, other flaws in decision making processes abound. Often times, key decision makers will attempt to use solely the power of their minds to sift through intricate details, never committing anything to paper. Others consider only the benefits of alternatives, seeking to maximize exclusively the positive aspects of the choice. Some may alternatively select options that merely minimize risks. And as in many business and government settings, political interests can enter the decision making process. Those higher in the chain-of-command can unjustly sway decisions one way or another.

The characteristics of complex decisions call for a more structured process; one capable of including intangible judgments. The Analytic Network Process (ANP) provides respite for the many flaws inherent to common decision making processes. In the course of the last four years, more than 150 complex decisions solved with ANP have been compiled. The following sections use those examples to illustrate basic characteristics of ANP. The need for cardinal preference is first discussed, followed by the need for structure. ANP structure is broken down into the positive and negative aspects of decisions, as well as the incorporation of complex interrelationships among criteria.

\section{Need for Cardinal Preference}

The proliferation of choices in today's marketplace has given individuals the added stress of choosing the best option among many. A problem immediately arises, because humans often have difficulty expressing their feelings through language and communication. When asked to rank order a list of items, individuals will generally be able to establish an ordinal preference. This means that the results of their ranking will show relative positioning among all options. This type of ranking lacks tangibility. There is no way to determine the magnitude of difference between each item. Although ordinal scaling may prove useful in some instances, there are obvious mathematical difficulties. For instance, operations beyond percentiles and summary statistics become meaningless due to the lack of an absolute zero reference point.

Marketers have realized the shortfalls of ordinal preference and have developed tools to address such issues. Conjoint analysis and other techniques exploit the inherent tradeoffs that one makes in selecting products to evoke useful preference data. Marketers are left with cardinal data that can show exactly which product attributes are favored and by what magnitude.

Decision makers must also consider these tradeoffs. The need for cardinal preference arises in almost every decision, but many tools and traditional processes have no way of measuring it. Again, this seems to be a logical extension from the nature of human communication. When faced with many options, we cannot effectively relay our intangible judgments into tangible preferences.

Fortunately, ANP easily addresses this issue. By providing a mathematical framework based on pairwise comparisons at the lowest level, cardinal preferences can be derived. Things that seem impossible to convey are suddenly quantified and factored into the decision making process. This powerful feature of ANP has been illustrated time and time again for decisions in business, government, public policy, and politics where intangible judgments are the norm. The following example illustrates the use of cardinal preference in making a complex decision.

In 2003, one firm faced the pressures of a competitive industry rivalry. Although business had been somewhat profitable, all indications showed a potential for outstanding performance. Naturally, fingers were pointed, and questions arose as to the suitability of current management to lead the organization. Every stakeholder had his own opinion and preference, but collectively, no one could gain enough power

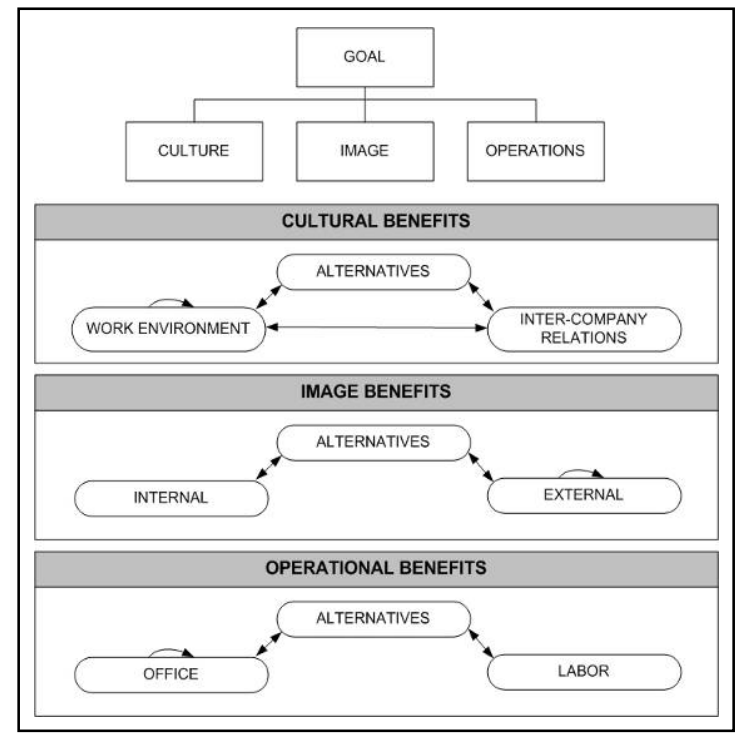

Figure 1: Benefit Control Criteria and Decision Networks 
to make the decision. An independent team was employed to use the ANP. This way, judgments could be broken down to their lowest levels to see the real impact a management replacement would have on business. Alternatives were given as “No Change," "Partial Replacement,” or "Complete Replacement.”

The independent team next developed BOCR networks to compare the alternatives. The networks contained control criteria, each with underlying sub-networks. Figure 1 shows the benefit control criteria and decision networks for this example. Within each decision network were various clusters, each with several nodes. For instance, the "Work Environment" cluster in the Cultural Benefits network contained the nodes of flexibility, teamwork, and motivation. Without the use of ANP, one may look at these three attributes and be able to determine how each would be affected by the three alternatives. The results may indicate that the "Complete Replacement" alternative provides the greatest flexibility, followed by "Partial Replacement," then "No Change." However, even though an ordinal ranking is possible, the magnitude of differences is neglected. Decision makers cannot be certain of how close the first and second place alternatives stand.

With ANP however, cardinal preference rankings are easy to attain. Decision makers are simply asked to rate the alternatives, two at a time, on how much flexibility one would achieve relative to the other. The process is repeated until all pairs are considered. Figure 2 illustrates the summary measurement data, which shows how the team gave magnitudes to the differences in the flexibility attribute. As shown, "Complete Replacement" offers a very strong (value of 8) flexibility compared to "No Change," and so forth. The ANP has effectively turned the ordinal preferences into tangible, cardinal ones.

\begin{tabular}{|c|c|c|c|c|c|c|c|c|c|c|c|c|c|c|c|c|c|c|}
\hline Complete Replacement & 9 & 8 & 7 & 6 & 5 & 4 & 3 & 2 & 1 & 2 & 3 & 4 & 5 & 6 & 7 & 8 & 9 & No Change \\
\hline Complete Replacement & 9 & 8 & 7 & 6 & 5 & 4 & 3 & 2 & 1 & 2 & 3 & 4 & 5 & 6 & 7 & 8 & 9 & Partial Replacement \\
\hline No Change & 9 & 8 & 7 & 6 & 5 & 4 & 3 & 2 & 1 & 2 & 3 & 4 & 5 & 6 & 7 & 8 & 9 & Partial Replacement \\
\hline
\end{tabular}

Figure 2: Comparisons With Respect to Flexibility in the Alternatives Node

Once in tangible form, such judgments are used in the ANP to quickly determine the most beneficial alternatives. Similar comparative ratings also relate the control criteria in each BOCR network. The independent team determined that a complete replacement of management was the best decision, given the relevant strategic criteria.

In addition to business examples, intangible judgments have been captured in areas such as politics, local, state, and federal government, the economy, public policy, sports, and more. The ability of determining cardinal preferences is essential to almost any decision, and thankfully, the ANP is based on this concept.

\section{Need for Structure}

The previous discussion illustrated how cardinal preference is fundamental to the ANP and to sound decision making. But simply being able to quantify intangible judgments will not ensure a good decision. A deeper structure must be used to pull all the essential elements of the decision together. Next, see how the ANP does just that by considering both positive and negative decision networks and by incorporating complex relationships into the process.

\subsection{Considering Both the Good and the Bad}

When decisions are processed in traditional manners, individuals are prone to certain errors. Many times, people will neglect some of the relevant components of the decision or focus only on those aspects that impact them personally. A trend has arisen in American culture that can be most appropriately called "spend now, justify later." When individuals are confronted with decisions, especially in the marketplace, they fail to consider costs and risks. They focus solely on the perceived benefit they will receive through the new product or service. This limited foresight results in skyrocketing amounts of personal debt that will come back to haunt these individuals in the future. It appears that when people are given mechanisms to bypass the short-term consequences of their decisions, in fact, those consequences do get completely neglected. 
This trend may be endemic to American culture on a personal level, but does it apply in the corporate world? Some recent consulting work may offer more evidence of its existence in other settings. An organization needed help determining customer preferences for a future internet based product. After a consultant conducted a focus group and survey, it became clear that users had very negative reactions to the online product. When the key decision maker for the organization was alerted to these results, she was quite upset. It suddenly became clear that the consultant's services were being used as a tool to justify a purchase the decision maker had already made. She had expected the results to confirm her decision to purchase the online platform, but when they didn't, she was stuck with the responsibility for a product that may have been a waste of money. In this case alone, poor decision making compromised tens of thousands of dollars.

Many past ANP examples illustrate this phenomenon. Looking through the compiled projects, seven of the first twenty projects considered had an overall result that differed from the perceived benefits. Thus, it appears quite common that the alternative holding the most benefit is not necessarily the one that should be selected. An example from 2004 illustrates this concept further.

A car manufacturer in Brazil was faced with the challenge of deciding how to overcome the high costs associated with running one of its main plants. Since the plant was located in a high-unionized region, it could not compete with plants in other areas. In addition, the decision to do anything would be subject to both government and community scrutiny. The ANP was used in order to add structure to the problem and find a reasonable solution. Alternatives were decided to be "Close the Facility," "Develop a New Product" (in order to keep the plant running), or "Reduce Facility Size."

After completing all steps of the ANP, the benefit network was synthesized with the results shown in Figure 3. Obviously, from this angle, the highest benefits were derived through plant closure. However, the structured steps of ANP forced the decision makers to consider more than just the benefits. Once the complete BOCR networks were synthesized, the overall outcome was quite different. As Figure 3 shows, the short-term solution would be to "Develop a New Product." The opportunities and lower costs/risks of this option made up for its lower perceived benefits.

\begin{tabular}{|c|c|c|c|}
\hline Alternative & Benefits & $\begin{array}{c}\text { Outcome } \\
\text { (BO/CR) }\end{array}$ & $\begin{array}{c}\text { Outcome } \\
\text { (Additive) }\end{array}$ \\
\hline Close the Facility & 1.0000 & 0.4755 & -0.8778 \\
\hline Develop a New Product & 0.5992 & 1.0000 & -0.4640 \\
\hline Reduce Facility Size & 0.4536 & 0.4845 & -1.0000 \\
\hline
\end{tabular}

Figure 3: Synthesized Results for Manufacturing Plant Shutdown

Another interesting finding from the model was that in the long-run, none of the options appear favorable, as seen in the last column of Figure 3. Although "Develop a New Product" is the least negative option, the decision should be further scrutinized. The decision makers must investigate even more alternatives in order to determine if favorable ones may exist. If not, developing a new product may provide the least costly alternative. Although the best long-term option is still unclear from this analysis, one thing is clear: the decision makers would have been in error had they selected the option with the most benefits.

The way perceived benefits can taint a decision is readily apparent from these and other examples. Yet another error plagues decision makers that may be more hidden. Often times, governments and businesses will be faced with decisions in which no alternatives appear very lucrative. When it comes to this point, they may select the alternative that presents the lowest amount of risk. The results of past ANP projects again show that this flaw can affect sound decision making.

An example from 2005 illustrates this shortcoming in detail. A unique situation had led to separate governments at the city and county level performing similar functions. Taxpayers eventually realized that the two governments could be more efficient as one, and thus public sentiment forced a committee to analyze the possible merger. In this situation and many other governmental decisions, the parties tend to consider the least risky options when making decisions. This occurs because first, and for good reason, it's normally taxpayer money. And second, because lower risk options usually avoid change, whereas more risky options lead to new processes and procedures that everyone has to learn. 
The committee arbitrarily considered four alternatives to solving the problem: "City Morph into County," "Do Nothing," "Full Merge," or "Services Only” (eliminate redundant services, but keep governments separate). When the committee used the ANP, an interesting result occurred. As Figure 4 shows, the "Do Nothing" option carried the lowest risk. In the past, the decision may have been made on this result alone. Since the risk of keeping the status quo was the lowest, that's the option the government would have selected. But the synthesis of risk alternatives only tells half the story. Upon further analysis, the overall outcomes shown in Figure 4 lead to a different solution. "Do Nothing" is no longer viable. In fact, its long-run solution is highly negative, and thus unfavorable. The "Full Merge" option is best for the short-run and is the only favorable solution in the long-run.

\begin{tabular}{|c|c|c|c|}
\hline Alternatives & Risks & $\begin{array}{c}\text { Outcome } \\
\text { (BO/CR) }\end{array}$ & $\begin{array}{c}\text { Outcome } \\
\text { (Additive) }\end{array}$ \\
\hline City Morph into County & 0.4771 & 0.7104 & -1.0000 \\
\hline Do Nothing & 0.4057 & 0.4069 & -0.7552 \\
\hline Full Merge & 1.0000 & 1.0000 & 0.5122 \\
\hline Services Only & 0.7505 & 0.4964 & -0.1053 \\
\hline
\end{tabular}

Figure 4: Synthesized Results for City-County Merger

The examples illustrated here show how ANP effectively incorporates both the good and bad aspects of decisions. Instead of focusing on solely the benefits or the risks, ANP considers all relevant criteria.

\subsection{Incorporating Complex Relationships}

In addition to simply incorporating positive and negative decision criteria, ANP has the power to consider many complex, interrelated situations. In traditional processes, relationships between alternatives and decision criteria may be neglected. It is very easy to focus one's attention on the problem at hand and forget to account for those possible interrelationships. Yet sometimes, without taking such relationships into account, the decision will be incomplete or inaccurate. Thus, ANP provides a very useful solution to this error. By allowing users to add inner and outer dependencies and feedback among clusters and nodes within the problem, ANP addresses the shortcoming of traditional processes.

A very complex example from 2004 shows the relevancy of incorporating complex relationships into decisions. A team from the US was concerned with the state of the nation's energy policy. They decided to use the ANP to create a model that could account for the many issues involved with energy security in the US. Alternatives were based on a report submitted to the Vice President of the US in 2001 and included: "Status Quo Approach," "Energy Independence Emphasis," "Complete Energy Independence," or "Comparative Advantage Approach." BOCR networks were created with a high degree of complexity. Shown in Figure 5 is the benefits sub-network, which illustrates the many control criteria and sub-criteria.

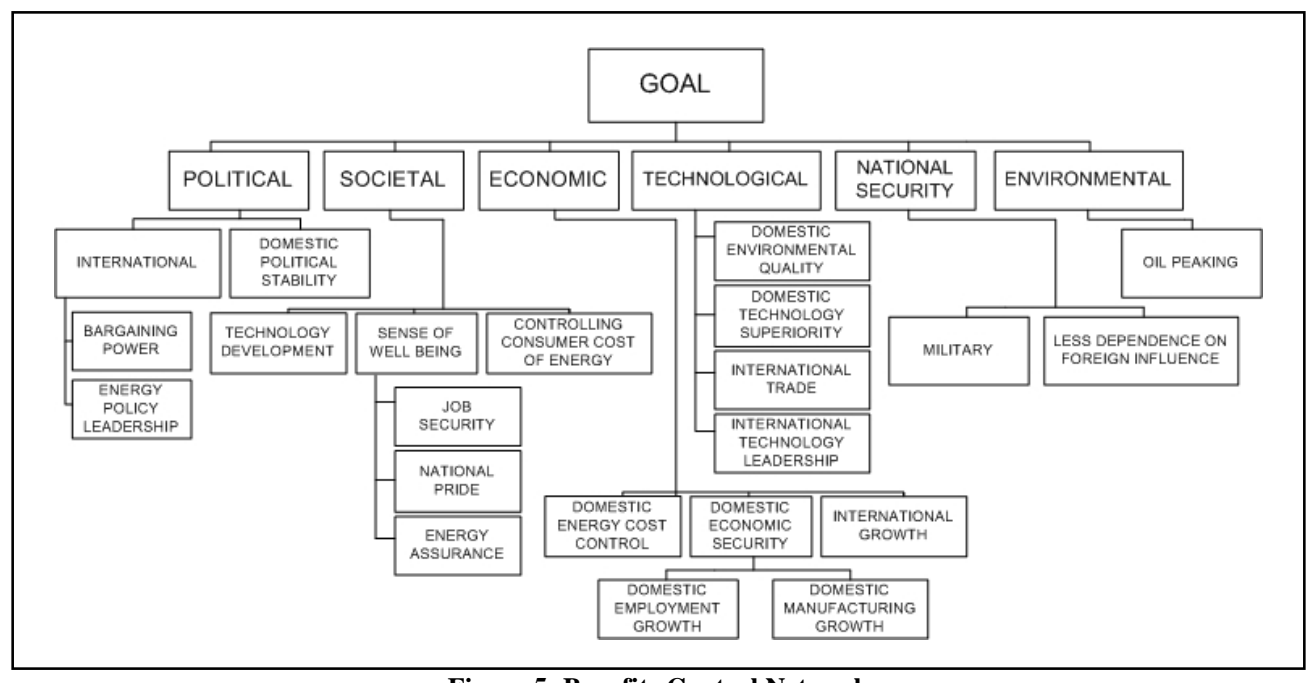

Figure 5: Benefits Control Network 
Within many of the sub-criteria of Figure 5 were further decision networks, as illustrated in Figure 6. The sheer degree of thought and planning that went into the creation of these networks is astounding. The team effectively accounted for the relationships within all stakeholder groups of the energy security decision.

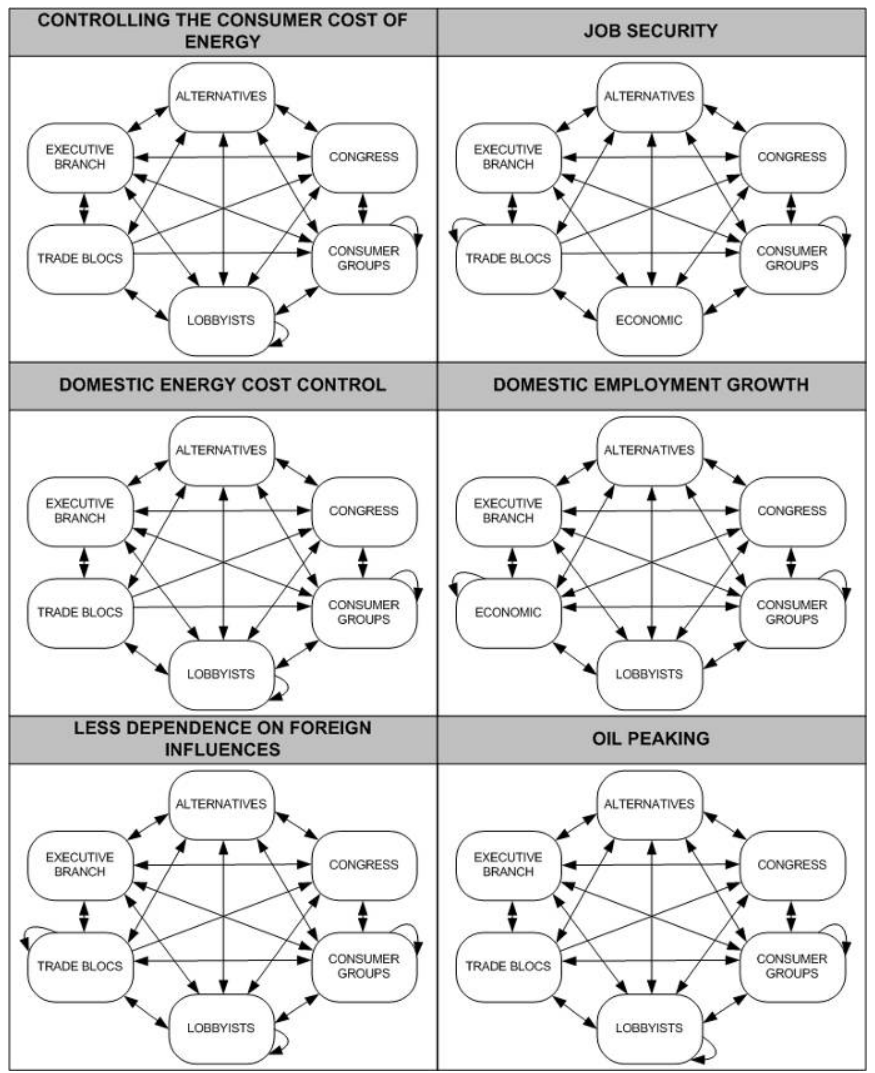

Figure 6: Decision Networks within Benefits Control Criteria

Whereas other decision processes may have led to inaccurate results, the ANP was able to consider all relevant relationships. The US energy security example is just one instance where complex decision networks more accurately reflect reality. By giving users the flexibility to create their own networks, the ANP allows for the incorporation of as much complexity as is necessary. Users should be glad to know that this freedom of design ultimately results in a more complete and sound decision.

\section{Conclusion}

With the various errors inherent to traditional decision making processes, a need has arisen for a better method. The Analytic Network Process effectively overcomes the flaws to which decision makers are prone. The fundamental structure of ANP allows it to generate cardinal preferences. This is significant because it adds tangibility to otherwise intangible judgments. In addition to establishing cardinal preference, the ANP adds a distinct structure to every problem. Users can create their own unique decision models, but several structural features of ANP point users to the correct outcome. First, the ANP incorporates both the good and the bad into decisions. Many examples show how traditional processes consider only benefits or only risks when seeking a solution. The ANP overcomes this shortfall by structuring networks into the BOCR framework. Secondly, the ANP allows for very complex decision networks. In the past, detailed intricacies and interrelationships were simply disregarded or forgotten. The ANP considers all dependencies and feedback relationships, so as to produce a more complete decision process.

In conclusion, the key features of cardinal preference, incorporation of positive and negative criteria, and integration of complex relationships give ANP a powerful approach to decision making. Many real world examples 
have shown how these fundamental and structural features have led to better decisions in the past and will continue to do so in the future. 